\title{
The Spectrum of Potassium excited during its Spontaneous Combination with Chlorine
}

\author{
BY \\ I. A. RAMDAS. \\ (Received for publication on the 14th June, 1928.)
}

\section{Abstract.}

The paper gives data concerning the spectrum of potassium excited while it burns spontaneously in an atmosphere of chlorine. The arc lines, an intense broad band in the red region (due to molecules of potassium) and a few feeble lines which appear to be the enhanced lines in the violet region are emitted.

The explanation of these results has been attempted in a general way and the possibility of thermal excitation has been considered.

\section{Introduction.}

The spectrum of ionised potassium has been extensively studied by various writers. Recently Foote, Meggers and Mohler ${ }^{1}$ have shown by a very simple method how the development of the spectrum of an element from its simple single line spectrum to its arc spectrum and finally into the more complicated enhanced spectrum could be studied and ex plained in detail. Their results prove that the very high potentials used by previous workers are not essential for exciting the various spectra of elements.

A Astro, Phyeical Journal, Vol, 55, \$. 145, (1922). 
The present writer 1 in a communication to "Nature" three years ago mentioned that the spectrum of the light emitted during the spontaneous combustion of pure potassium in chlorine showed in addition to the arc lines and the emission band in the red due to the molecules, some feeble enhanced lines. Recently the experiments were repeated during the writer's visit to Calcutta and the results obtained confirm the previous observations. The exciting agent in the present instance is chlorine, the electron affinity of which is five volts and is sufficient to excite at least the arc spectra of potassium, rubidium and caesium, but not effectively of sodium whose ionising potential is 5.13 volts. Potassium burns with a brilliant flame and rubidium even more vigorously but a piece of sodium, even when heated, failed to combine with chlorine with appreciable light emission. We may, of course, expect fluorine to excite sodium vigorously. The results obtained in the case of potassium and chlorine are discussed in the following section.

\section{Experimental Procedure and Results.}

In the experiments Merck's potassium was used. After removing the kerosme vapour and the scum by heating suitable quantities in an iron ladle, the pure metal was poured off on a dry glass plate and small pieces were cut for use after it had solidified. The chlorine gas was prepared by the action of hydrochloric acid on potassium permanganate. The gas was purified by passing through water, oopper sulphate solution and water again and collected in a quartz flask. When a small piece of potassium kept in a deflagrating spoon made of iron was lowered into the flask, the potassium instantly burst inte a brilliant flame which lasted for a few seconds filling the whole tube with a cloud of potassium chloride.

For photographing the spectrum of the chemi-lumines cence, about a dozen such ignitions with the flask in front of 
the slit of the smaller type of Hilger quartz spectrograph were found sufficient when Ilford Panchromatic plates were used. The ignitions always took place successfully provided the surface of the potassium used was quite fresh and the builb was filled completely with chlorine.

The photographic plate showed, on visual examination, besides the band in the red and the comparatively mure intense arc lines, the sharp forbidden line at 4642 and four or five very feeble but distinct lines in the region 4642 to 4045 . Owing to the super-position of the continuous spectrum in this region, these feeble lines could not be easily located through the travelling microscope, but fairly reliable measurements cuuld be obtained.

These were later verified from the measurements of a micro-photometer (self-recording) record of the plate. See Fig. 1. The various lines measured are listed in the table

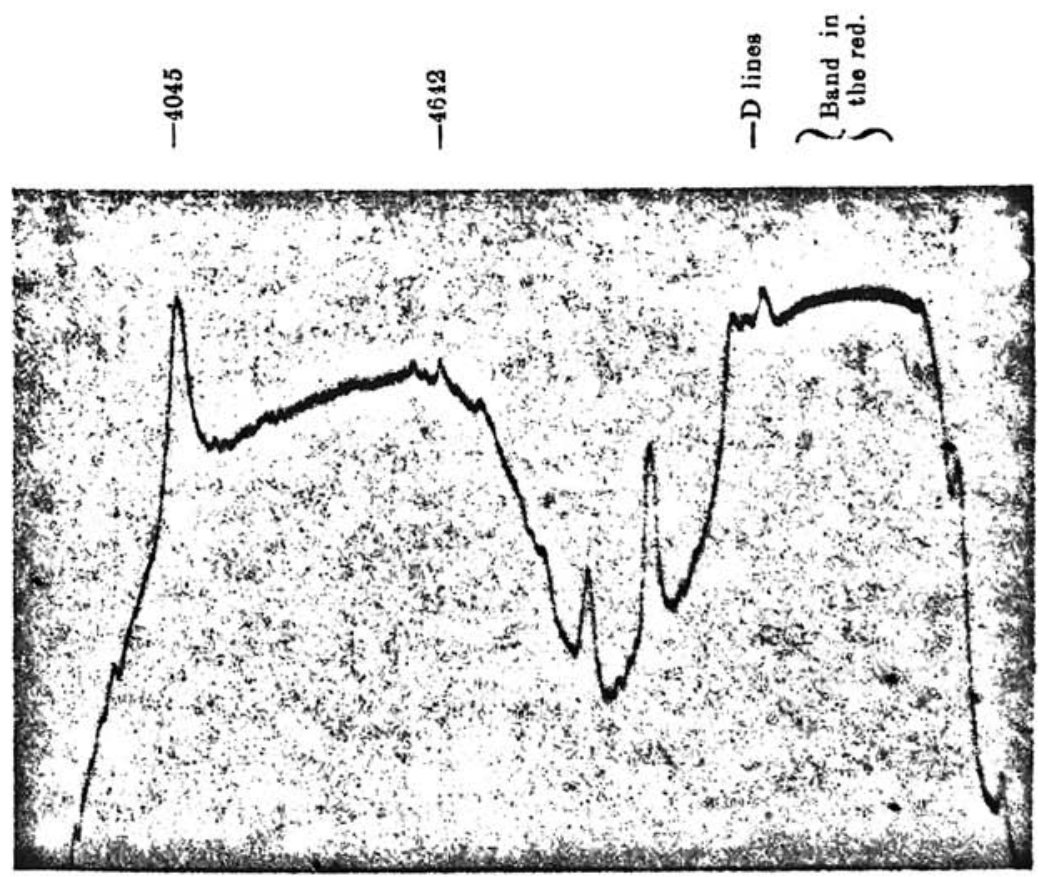

F'ro. 1. 
below Owing to small dispersion combined with the special eircumstances in which the lines are excited (comparatively high pressure, i.e., more than one atmosphere) most of the doublets were unresolved and unusually broad, although the slit of the spectrograph was kept quite harrow. The fainter lines could not be reproduced in print.

TABLE.

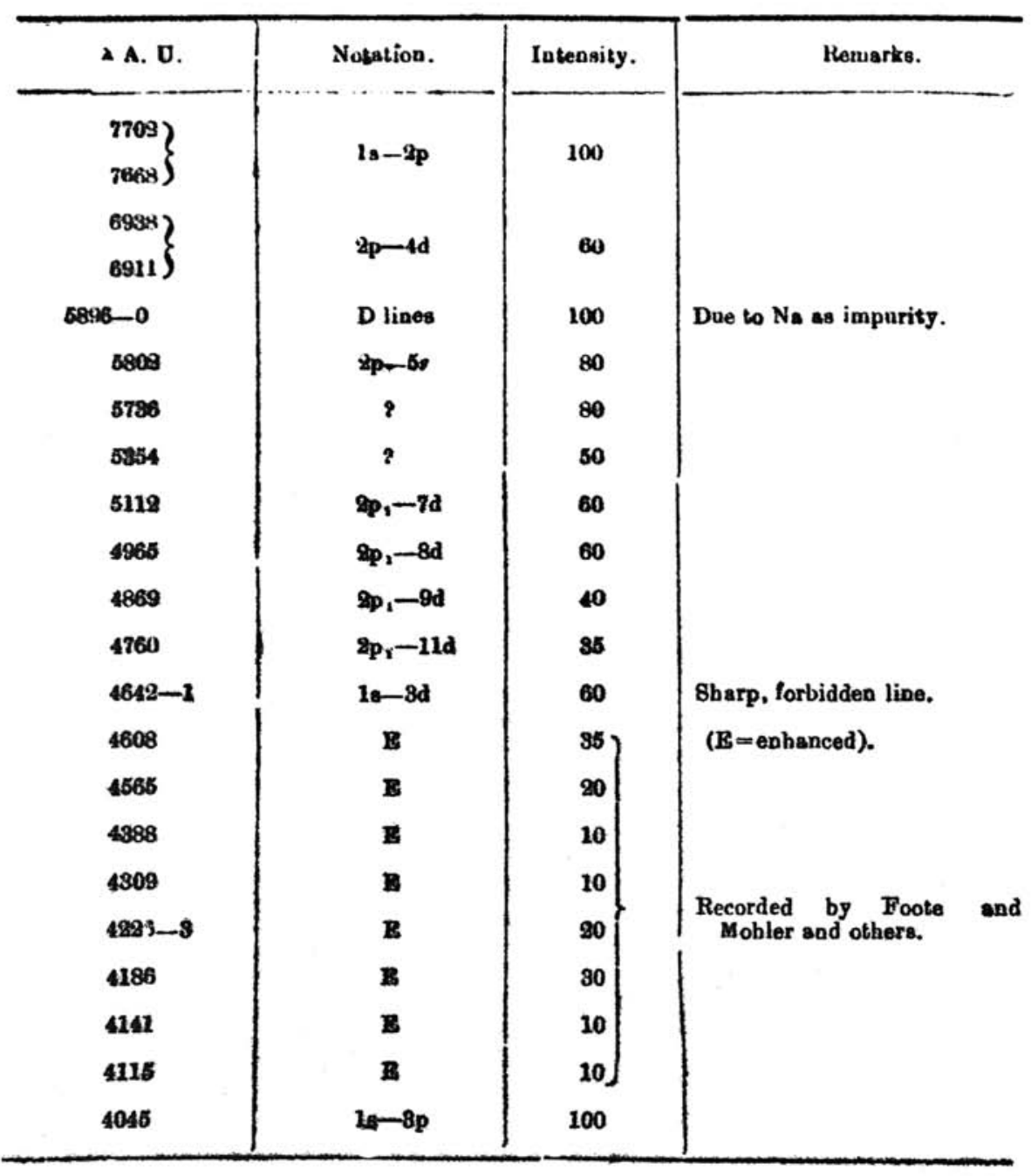


3. Discussion of Results.

The observed spectra are :

(i) the band spectrum $\quad$... $\quad$ most intense

(ii) arc spectrum $\quad$... less intense

(iii) enhanced spectrum $\quad \ldots \quad$ least intense.

These require the presence of $\mathrm{K}^{+}$and free electrons, $\mathrm{K}^{++}$ or at least $\mathrm{K}$ with one of its $\mathrm{M}$ electrons completely removed and the valence electron in one of the onter orbits, and $K$ molecules. The voltage required to excite the arc and enhanced spectra, are $4 \cdot 1$ and 23 volts respectively. If due to temperature excitation alone, the last would require temperatures of the order of $12,000^{\circ} \mathrm{A}$, while simple ionisation would require $6000^{\circ} \mathrm{A}$. We shall briefly consider the possibility of attaining such high temperatures in the reaction.

Following 1Fchardson's method ${ }^{1}$ one may work out the value of ' $\mathrm{T}$ ' in the ruaction. In general, if $\mathrm{H}$ is the heat available in gram calories, per gram molecule, we shall have as an upper limit for the temperature $T, \frac{3}{2} k T=\frac{J H}{N}$, when $k$ is Boltzmann's constant $1.346 \times 10^{-10}, J$ is the mechanical equivalent of heat, $4 \cdot 154 \times 10^{\prime}$ and $\mathrm{N}$ is the number of molecules per gram molecule, $6.2 \times 10^{2}$

In our case, the reaction of the following type can take place (though finally all the potassitum is converted into $\mathrm{KCl}$ )

(i) $2 \mathrm{~K}+\mathrm{Cl}_{2} \longrightarrow 2 \mathrm{KCl}+208 \times 10^{\circ} \mathrm{gr}$. cals.

(ii) $2 \mathrm{~K}+\mathrm{Cl}, \longrightarrow \mathrm{KCl}+\mathrm{K}^{+}+\mathrm{Cl}+$ electron

$$
+104 \times 10^{3} \mathrm{gr} \text {. onls. }
$$

$\left(104 \times 10^{8} \mathrm{gracal}\right.$. is onfy an upper limit in this case.)

(iii) $\mathrm{K}^{+}+\mathrm{Cl} \longrightarrow \mathrm{KCl}+163 \times 10^{s}$ gr. enls.

- Richardson, Phil. Trana. Roy. Boo. A. Vo. \$2, pp. 1-43, (1922). 
Assuming that in each case the total number of entities sharing the energy liberated is equal to the totai number of atoms on the left hand side of the equations plus an electron in each case, we get the following value of ' $\mathrm{T}$ ':

$$
\begin{aligned}
& \text { (i) } \mathrm{T}=14,0100^{\circ} \mathrm{A} \\
& \text { (ii) } \mathrm{T}=7,0010^{\circ} \mathrm{A} \\
& \text { (iii) } \mathrm{T}=18,700^{\circ} \mathrm{A} .
\end{aligned}
$$

It is not unlikely that there may be zones in the flame developed during the reaction corresponding roughly to the above temperatures, just as there are zones of different temperatures in the electric arc. At any rate, there is nothing to prevent the existence of local and momentary flashes of very high temperatures inside the flame though any part of the flame may not attain steady temperatures of the above order. If this possibility is accepted, there is no difficulty in accounting for the appearance of the feoble enbanced lines in the spectrum of the chemi-luminescence.

The investigation was oarried out at the Indian Association for the Cultivation of Science, and the author's best thanks are due to Prof. C. V. Raman, for providing the necessary facilities. 